\title{
Age-related Control Strategies to Execute the First Step from Standing in Older Adults
}

\author{
Mansoo Ko ${ }^{*}{ }^{1}$, Matthew Cole ${ }^{2}$, Rachel Vinyard ${ }^{2}$ \\ ${ }^{*}$ University of Texas Medical Branch, USA \\ ${ }^{2}$ Angelo State University, USA
}

Purpose The purpose of this study was to identify the effect of aging on the dynamic balance of gait initiation (GI) between healthy elderly adults and young adults. Methods This study used descriptive analysis. Total 17 subjects, 8 healthy elderly and 9 young adults were recruited from the local community, San Angelo, Texas. EMG sensors (Biometrics Ltd., Ladysmith, VA, USA) were attached to the TA and Sol muscles bilaterally to measure peak amplitude and time to peak amplitude. Subjects were instructed to stand comfortably while barefoot on the High Resolution (HR) mat (Tekscan Inc., South Boston, MA, USA) to assess 1) COP anterior-posterior excursion, 2) COP medial-lateral excursion, 3) COP area, 3) COP backward motion distance, 4) COP motion to swing limb distance. The subjects were then given a verbal cue to start walking from a quiet standing. Results The healthy young group illustrated greater lateral and backward movement of COP as well as COP A-P and L-R excursion than the elderly group. In addition, the healthy young group showed greater peak amplitude of bilateral TA muscles during GI. Time to peak TA muscle amplitude was much faster in the healthy young group than the elderly group during GI. Conclusion The limited dynamic balance observed in the elderly group compared to healthy young adults during the task of GI. This finding suggests that sway analysis during GI could be used as a screening tool to identify the impaired dynamic balance for people who present balance deficits.

Key words Age-related control, older adults, dynamic balance, gait initiation, EMG

Corresponding author Mansoo Ko (mako@utmb.edu)

Received date 20 January 2018

Revised date 9 February 2018

Accepted date 21 February 2018

\section{Introduction}

Gait initiation (GI) is defined as a transition between standing and walking. ${ }^{1)}$ This task has been used as one example of motor control in which a motor program is used precisely to execute a task ${ }^{1)}$ because GI is a centrally-organized motor program model uninfluenced by peripheral feedback. Thus, GI has stereotyped neuromotor processes. ${ }^{1,2)}$ For healthy people, the task of GI begins with inhibition of tonic soleus (Sol) activity followed by onset of the tibialis anterior (TA) of both the swing and stance limbs. Swing limb gluteus medius (GM) is also active at this time. ${ }^{1)}$ This muscle sequencing pattern is responsible for the move-

doi : http:dx.doi.org/10.17817/2018.01.24.111235 ment of the center of pressure (CoP) backward and toward the swing limb, which tends to propel the CoP forwards and towards the stance limb (1). Brunt et al. (1991) identified the invariance of the relative timing of onset of muscle activity, swing toe-off, and heel strike based on EMG and force plate recordings during GI. ${ }^{3)}$ Removal of sensory feedback by a tibial nerve block did not influence certain temporal parameters of GI. ${ }^{4)}$ Crenna and Frigo (1991) also found that during GI the relative timing between Sol inhibition and TA activation is invariant." Accordingly, GI provides an optimized motion environment to evaluate neurological changes or deficits in motor control following stroke because the pre-programmed task is independent of peripheral influence prior to movement. ${ }^{3)}$

GI has usually been divided into two phases. ${ }^{1,6-9)}$ 
One is a preparation phase ${ }^{1)}$; the other is a step execution phase. ${ }^{1)}$ However, studies have provided different criteria to determine the first or second phase of GI. Breniere et al. (1987) suggested that GI is composed of two phases based on the heel-off of the swing limb ${ }^{1,10)}$ because the forward velocity of CoP rapidly increases after swing heel-off. ${ }^{3,10)}$ In particular, Breniere and his colleagues indicated that faster gait required a longer first phase and a shorter second phase of GI. ${ }^{11,12)}$ Jian et al. (1993) indicated the first phase of GI ends with the toe-off of swing limb. ${ }^{1,9)}$ This first phase is responsible for the movement of the CoP backward and toward the swing limb, which tends to propel the CoP forwards and towards the stance limb. ${ }^{1)}$ In the second phase, the CoP moves forward fast to reach the steady-state walking while CoP moves across to the stance limb.9) Brunt et al. (1999) proposed that the first phase of GI is determined by time to heel-off on the stance limb for two reasons. First, the time to stance limb heel-off remained invariant for GI at fast and self-selected walking speeds and for obstacle crossing tasks. Second, the programming of GI prior to stance limb heel-off (first phase) is independent of the programming of GI after stance limb heel-off (second phase) because the acceleration force in stepping obstacle crossing was significantly greater than GI across self-paced and fast initiation. $^{1)}$

Therefore, the purpose of this study was to identify the effect of aging on the dynamic balance of GI between healthy elderly adults and young adults. Specifically, this study investigated 1) the CoP motion between two lower limbs and 2) time to peak amplitude on TA muscle using electromyography (EMG) in healthy elderly adults compared to young adults during GI.

\section{Materials and Methods}

\section{Subjects}

This study was a between group design that used nine healthy young adults ( 7 female, 2 male) recruited from the San Angelo local community, USA. Healthy young adults were excluded if they had any neurological or orthopedic problems. Eight ( 7 female, 1 male) healthy elderly subjects were recruited from the San Angelo Community through the San Angelo Spring Fling. At the spring fling potential subjects were initially screened by age, past medical history and the use of the BERG Balance Test. Healthy Elderly subjects were excluded if they were less than 60 years of age, had any neurological, balance disorders or orthopedic problems. Gender matching was attempted; however, we were unable to find a second elderly male meeting all inclusion exclusion criteria. The average age of the healthy elderly group was 72.9 years old and the average age of the healthy young adults was 25.5 years old. This study was conducted with IRB approval.

\section{Procedures}

Subjects were instructed to wear comfortable clothes that provide access to the skin of their lower limbs. The skin was prepped with an alcohol pad to clean the skin that the EMG sensors would be placed on. Foot dominance was determined by asking each subject which leg they would use to kick a ball. All subjects reported to be right foot dominant. EMG sensors were attached to the Sol and TA bilaterally. Subjects were instructed to stand comfortably while barefoot on the Tek-Scan ${ }^{\circledR}$ HR mat. The subjects were then given a verbal cue to start walking and to continue walking 10 feet. After both feet had left the Tek-Scan ${ }^{\circledR}$ HR mat data collection stopped. Subjects had one initial practice trial and then each subject performed 3 trials of GI that were recorded. Stance width and step length were not preset.

\section{Data Analysis}

Prior to parametric tests, the Shapiro - Wilk test will be used to determine normality of all initial measures of the Tek-Scan and EMG data during GI trials for all the participants. Levene's test for equality of variance will also be conducted to check the basic assumption of parametric test. Due to the small sample size, the data did not stratified with the normality and homogeneity. Therefore, this study used descriptive analysis. The 
following parameters were extracted from the Tek-Scan ${ }^{\circledR}$ data: center of pressure anterior-posterior excursion (CoP A-P Exc), center of pressure medial-lateral excursion (CoP M-L Exc), center of pressure area (CoP Area). Also used were center of pressure backward motion distance (CoP Bwd), center of pressure motion to swing limb distance (CoP SWG), duration of time between GI and toe off of the swing limb (TSW) and the duration of time between GI and toe off of the stance limb (TST). The following parameters were extracted from the Biometrics Ltd. EMG: amplitude of peak muscle contraction and rates of force generation.

\section{Results}

Both the healthy elderly and healthy young adult groups displayed their CoP first moving posteriorlateraly (right) towards their swing foot (dominant/right foot) and then forwards towards their stance foot. However, when comparing the healthy elderly adults to the healthy young adults, the young adults CoP's traveled farther backwards and more laterally towards the swing foot as seen in Figure.1.

The young adult's CoP areas where also larger, indicating that their CoP trajectories' covered more area, as indicated by the red area circle compared to the green area circle in Figure 1.

The young adults CoP's also had larger A-P exc and M-L exc, indicating their CoP's traveled more distance in the anterior-posterior plane (Figure 2) and in the medial-lateral plane (Figure 3). The young adults' peaks are higher and lower, indicating the CoP for the young group traveled farther. The young adult group had steeper slopes than then healthy elderly adults, indicating the distance traveled by their CoP, occurred more quickly than the healthy elderly adults.

When comparing the duration of time from GI to toe off of the swing limb and stance limb between the healthy elderly subjects to the healthy young adults, the healthy elderly group had slower times. Table 1 summarizes the Tek-Scan ${ }^{\circledR}$ CoP data. The EMG results illustrated differences in both force and speed of the
(Standard deviation)

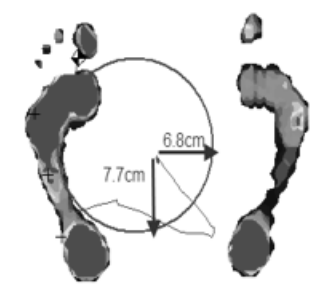

Young

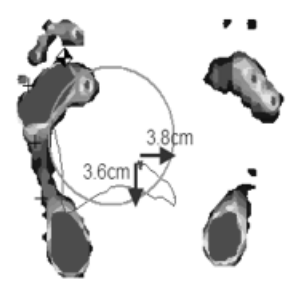

Elderly
Figure 1. Cop movement between young and elderly people

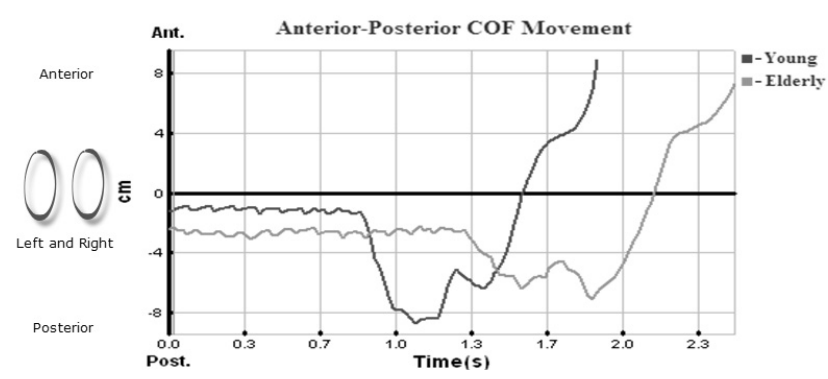

Figure 2. Anterior Posterior CoP movement

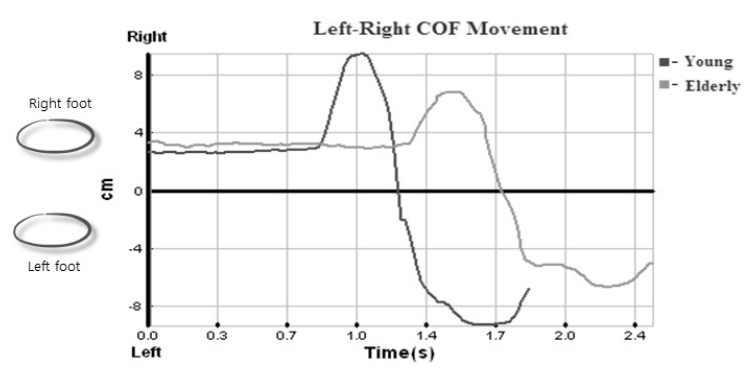

Figure 3. Medial Lateral CoP movement

contraction when comparing the two groups. Though both groups illustrated inhibition of the tonic Sol and contraction of bilateral TA, see figure 4 , the younger group experienced a stronger contraction in all muscles except for the swing Sol. The younger group also experienced a much faster rate of force generation, the amount of time from initiation to the peak amplitude of the initial contraction, in bilateral TA. 


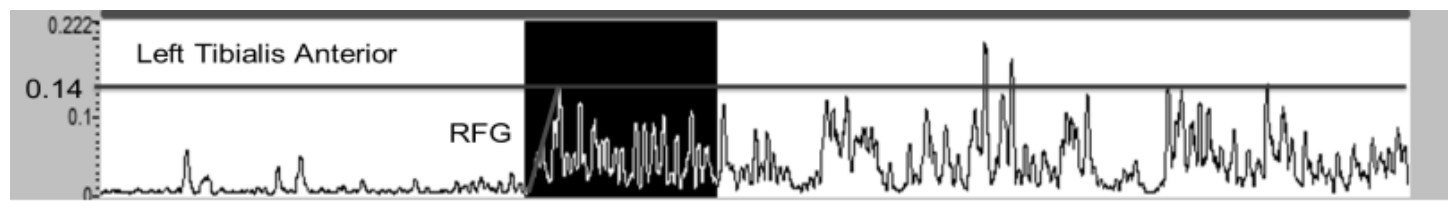

Older adults

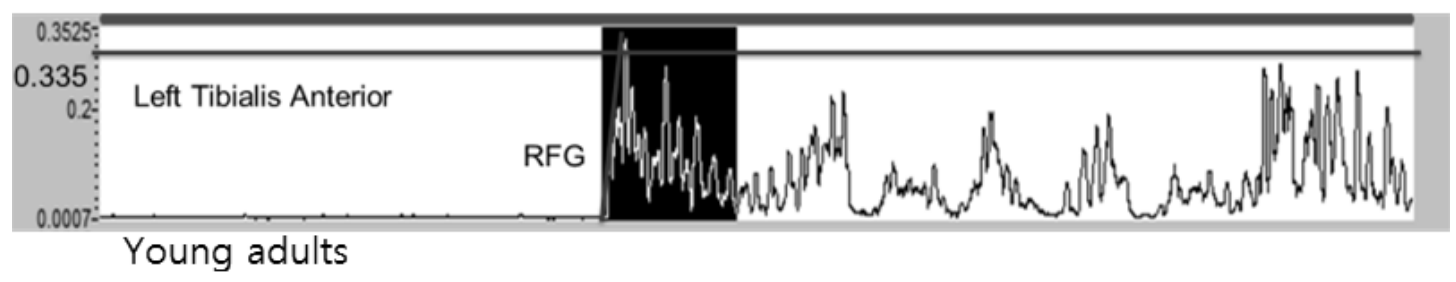

Figure 4. Rate of force generation and peak amplitude (older vs young adults)

Table 1. Tekscan Parameters: Mean

\begin{tabular}{ccc}
\hline Outcome Variables & Young & Elderly \\
CoP A-P Exc (cm) & $16.89(1.63)$ & $15.19(1.35)$ \\
CoP L-M Exc (cm) & $14.05(2.54)$ & $13.10(1.53)$ \\
CoP Area $\left(\mathrm{cm}^{2}\right)$ & $158.69(32.25)$ & $133.65(24.96)$ \\
CoP Swg (cm) & $4.11(1.20)$ & $3.96(0.94)$ \\
CoP Bwd (cm) & $4.43(0.96)$ & $3.55(1.01)$ \\
TSW (secs) & $0.56(0.11)$ & $0.59(0.09)$ \\
TST (secs) & $1.19(0.08)$ & $1.25(0.12)$ \\
\hline
\end{tabular}

\section{Discussion}

Aging can lead to lifestyle changes such as increased obesity and reduced fitness status, which could be due to general de-conditioning, muscle weakness, decreased joint mobility, and compromised peripheral sensory information. Those motor and sensory deficiencies in older adults can further result in kinematic alternations in gait, postural instability, and an increased risk of falling. Therefore, the purpose of this study was to identify the effect of aging on the dynamic balance of gait initiation (GI) in older adults.

In 1999, Chang and Krebs attempted to determine whether measurements of center of gravity-center of pressure separation (CG-CP moment arm) during GI can differentiate healthy from disabled subjects with sufficient specificity and sensitivity to be useful as a screening test for dynamic balance in elderly patients. ${ }^{13)}$ By comparing three groups, healthy elderly, disabled elderly, and vestibular hypofunction elderly, they determined that peak CG-CP were significantly different between groups and that this sort of screening tool is a valid way to identify people with poor balance. This study determined that there are differences between elderly people and young people with regards to movement of CoP. Based on Chang and Krebs' data, this would suggest that the elderly group has decreased balance and an increased fall risk.

Polcyn et al 1998, determined that the central nervous system uses stable and efficient mechanisms for dealing with the inherent instability involved with GI and that the integrity of these mechanisms degrade with age.2) This study echoed this determination by finding that the $\mathrm{CoP}$ of older adults moves less posterolaterally toward the swing limb at a lower speed when compared to young adults. Polcyn et al also determined that though soleus inhibition prior to TA activation is present in both older and young adults, this pattern is seen less frequently in the older population. ${ }^{2}$ This study also found differences in muscle activation. When compared to healthy young adults, healthy older adults experience smaller peak contraction in both TAs and the standing soleus. The younger group also experienced a faster rate of first generation of TA bilaterally. This study simultaneously captured evidence of decreased muscle performance in the elderly group in the form of decreased peak amplitude and 
slower rate of force generation. This study hypothesize that this decreased muscle performance is directly related to the smaller movement of $\mathrm{CoP}$ in the elderly group. Therefore, training these specific muscle groups to increase muscle performance could increase the area of $\mathrm{CoP}$ in the elderly group, improving dynamic balance, allowing more a more natural GI pattern, and decreasing fall risk.

In conclusion, the older adults illustrated significantly limited backward movement of CoP (older vs young: $3.55 \mathrm{~cm}$ vs $4.43 \mathrm{~cm}$ ) with reduced and slow rate of force generation of bilateral TA muscles compared to healthy young adults during GI. This finding suggests that sway analysis during GI could be used as a screening tool to identify the impaired control strategies related to dynamic balance in older adults.

\section{References}

1. Brunt D, Liu SM, Trimble M, et al. Principles underlying the organization of movement initiation from quiet stance. Gait Posture. 1999;10(2):121-8.

2. Polcyn AF, Lipsitz LA, Kerrigan DC, et al. Age-related changes in the initiation of gait: degradation of central mechanisms for momentum generation. Arch Phys Med Rehabil. 1998;79(12):1582-9.

3. Brunt D, Lafferty MJ, McKeon A, et al. Invariant characteristics of gait initiation. Am J Phys Med Rehabil. 1991;70(4):206-12.

4. Fiolkowski P, Brunt D, Bishop M, et al. Does postural instability affect the initiation of human gait? Neurosci Lett. 2002;323(3):167-70.

5. Crenna P, Frigo C. A motor programme for the initiation of forward-oriented movements in humans. J Physiol. 1991;437:635-53.

6. Breniere Y, Do MC. When and how does steady state gait movement induced from upright posture begin? J Biomech. 1986;19(12):1035-40.

7. Breniere Y, Bril B, Fontaine R. Analysis of the transition from upright stance to steady state locomotion in children with under 200 days of autonomous walking. J Mot Behav. 1989;21(1):20-37.

8. Breniere Y, Do MC. Control of gait initiation. J Mot Behav. 1991;23(4):235-40.

9. Jian Y, Winter, DA, Ishac, et al. Trajectory of the body COG and COP during initiation and termination of gait. Gait Posture. 1993;1:9-22.

10. Breniere Y, Do MC. Postural modifications during the 1st step of walking. Journal De Biophysique Et De Biomecanique. 1987;11(4):161-7.

11. Couillandre A, Breniere Y, Maton B. Is human gait initiation program affected by a reduction of the postural basis? Neuroscience Letters. 2000;285(2):150-4.

12. Lepers R, Breniere Y. The role of anticipatory postural adjustments and gravity in gait initiation. Exp Brain Res. 1995;107(1):118-24.

13. Chang H, Krebs DE. Dynamic balance control in elders: gait initiation assessment as a screening tool. Arch Phys Med Rehabil. 1999;80(5):490-4. 